\title{
Audio-visuel et formation permanente : un outil transculturel pour l'apprentissage de la gestion par des dirigeants de petites entreprises
}

Marie-France Kouloumdjian et Louis Armellino

\section{(2) OpenEdition}

Journals

Édition électronique

URL : https://journals.openedition.org/edc/2985

DOI : 10.4000/edc.2985

ISSN : 2101-0366

Éditeur

Université de Lille

\section{Édition imprimée}

Date de publication : 1 mai 1987

Pagination : 191-204

ISSN : $1270-6841$

\section{Référence électronique}

Marie-France Kouloumdjian et Louis Armellino, «Audio-visuel et formation permanente : un outil transculturel pour l'apprentissage de la gestion par des dirigeants de petites entreprises », Études de communication [En ligne], 9 | 1987, mis en ligne le 24 février 2012, consulté le 21 septembre 2021. URL : http://journals.openedition.org/edc/2985; DOI : https://doi.org/10.4000/edc.2985

Ce document a été généré automatiquement le 21 septembre 2021.

(c) Tous droits réservés 


\title{
Audio-visuel et formation permanente : un outil transculturel pour l'apprentissage de la gestion par des dirigeants de petites entreprises
}

\author{
Marie-France Kouloumdjian et Louis Armellino
}

\section{De l'économie avant toute chose}

1 A la fin de la seconde guerre mondiale, la reconstruction du tissu industriel européen passe par la notion "d'économie de grande échelle » liée à l'importance des besoins à satisfaire. Chaînes de production, cadences de travail, sur le modèle nord-américain, donnent naissance à des groupes d'entreprises de plus en plus gigantesques (conglomérats). C'est à partir des années soixante que commence à se développer aux États-Unis, un mouvement qui tend à démontrer l'inefficacité de telles structures, particulièrement dans leur fonctionnement social; à l'inverse, il affirme le caractère humain des petites organisations, leur adaptabilité aux aléas des marchés, leur capacité de profits liée à leurs faibles coûts de gestion et enfin leur potentiel de réussite par l'esprit d'équipe qui les anime.

2 Dans le milieu des années soixante-dix, face à la concurrence des autres nations industrialisées, équipées pour des marchés mondiaux, la France tente de réagir en encourageant la petite entreprise : aides financières, primes, détaxations diverses incitent à la création. Mais leur multiplication entraîne un accroissement de la concurrence. Il y a parfois difficulté à survivre. Il ne suffit pas d'être bon, voire excellent technicien pour réussir, il faut être " gestionnaire » car les entreprises qui triomphent sont celles dont les ressources sont le mieux utilisées pour une rentabilité optimale, c'est la loi de l'économie du marché. 


\section{Une organisation sociale insaisissable}

Pourtant aucune définition objective ne caractérise de manière définitive la petite entreprise. Ni le chiffre d'affaires, ni le nombre de clients, le volume des stocks ou le niveau du bilan. L'appartenance au syndicat patronal (C.G.P.M.E.) est le fait du dirigeant à titre personnel et non de l'entreprise. Il y a cependant, une limite : moins de onze salariés (quinze avec les auxiliaires familiaux) pour l'entreprise artisanale. Au-dessus, rien sauf l'obligation de représentation syndicale et salariale pour plus de cinquante salariés. La règlementation fiscale reconnaît à la petite entreprise le régime du forfait pour un chiffre d'affaires maximal, mais tend à le faire disparaître ${ }^{1}$.

4 Comment alors la définir? Nous pensons, avec d'autres, qu'il y a petite entreprise tant que son dirigeant participe à la production, sans activité principale de gestion. Il s'ensuit que la structure est le plus souvent de type «solaire » au sens symbolique du terme : toute action est ramenée à la seule volonté du dirigeant, omniprésent, sans intervention véritable de l'encadrement. L'organisation est basée sur la production (biens ou services) avec la coopération d'un comptable plus ou moins gestionnaire. Le fonctionnement enfin, est ordonné autour de la production basée sur l'amalgame entre souhaits de la clientèle et compétences principalement techniques du dirigeant.

C'est une approche qui peut sembler fragmentaire parce que simplifiée, mais nous pensons qu'elle pointe les caractères propres à ce type d'organisme.

\section{Le dirigeant de petite entreprise : quel habitus ?}

5 Il pourrait paraître présomptueux et réducteur de vouloir brosser un portrait typologique global de ce responsable, alors que nous avons à peine pu définir la petite entreprise ; mais il nous a semblé possible de le tenter partiellement d'après notre expérience pour mettre, en particulier, en relief son attitude vis-à-vis de la gestion financière de l'entreprise.

De ce point de vue, nous avons repéré quatre modèles principaux de comportements chez les dirigeants.

\subsection{Tradition oblige}

7 On l'observe en général chez le successeur du dirigeant précédent (filiation ou désignation du compagnon en qui le patron se reconnaît le mieux, et qu'il façonne durant des années à l'entreprise). Le continuateur en perpétue les rites et le style de décision. Il se suffit de la situation, bloque toute possibilité de développement et prépare de même façon sa succession.

De formation technique (CAP, BTS, DUT), il se perfectionne peu, prétendant trouver dans ses acquis la réponse à toutes les questions. L'organisation est maintenue, bien que vieillotte, les incidents nombreux réclament l'attention du dirigeant, même pour des vétilles.

9 Les résultats de l'exploitation se dégradent alors, engageant une procédure de tassement, un jour, fatale : investissements rares, réduction des frais (tous!), faveurs coûteuses aux clients pour les conserver. C'est le comptable qui connaît le contenu du «tiroir-caisse »; il y a peu de gestion véritable. 


\subsection{De l'avant, toujours de l'avant}

10 travail, ce dirigeant investit, modernise, trouve de nouveaux créneaux de marché et, avec l'expansion, crée des profits. De formation technique moyenne, il ne manque aucun stage sur les technologies nouvelles, de sa spécialité à l'informatique. Il lit les magazines et revues spécialisés en les annotant. Boulimique du savoir, il est l'illustration de la formation continuée, jamais arrêtée. Il maintient une structure autoritaire, pille les bonnes idées autour de lui, soude l'équipe, a un second mais ne délègue jamais vraiment le pouvoir. L'organisation est renouvelée, flexible, modifiable suivant les objectifs à atteindre, ce qui le fait paraitre fantasque, instable à son entourage. L'équipe évolue en évacuant les hommes statiques au profit de ceux qui progressent, les clients sont comblés par le rapport prix/satisfaction et font la publicité eux-mêmes.

11 Il faut maîtriser l'expansion, et le comptable prépare les dossiers, commente les options proposées. Le dirigeant traduit en termes de production et un dialogue difficile s'engage car l'un parle financement et l'autre technique. La décision est prise par le dirigeant, plus souvent par intuition ou expérience que par raisonnement explicite.

\subsection{Le clonage technologique}

12 Patron de son unité, un cadre d'entreprise ressent, tout au long des années, la frustration de celui qui a un pouvoir et non l'autorité. Les conflits engendrés aboutissent à son départ et, regroupant ses collègues dans le même cas, il crée une entreprise où chacun apporte ses moyens financiers et sa qualification technologique. L'activité se déroule rarement dans un contexte différent de celui connu jusque-là, la gamme des produits reste identique, avec cependant, parfois, des méthodes de production améliorées. La formation de l'ingénieur marque fortement ce type de comportement; le dirigeant continue à apprendre dans sa technique, focalise ses efforts, se forme dans des cours, séminaires professionnels où il échange, en gardant toutefois ses secrets pour rester le meilleur. Il incite ses collaborateurs à créer leurs propres procédures de travail, et n'hésite pas devant la concertation: chacun met ses qualifications propres au service de tous. Le dirigeant définit les politiques et coordonne les actions ; la productivité croît grâce aux équipements de qualité, ordre et méthode facilitent la communication et le travail de groupe. L'ambiance est agréable, nul ne refuse l'effort pour un nouveau travail. La qualité du standard ou d'un prototype témoigne d'un sérieux remarqué.

13 Les investissements onéreux sont suivis de près par le comptable-gestionnaire qui, à intervalles réguliers, annonce les résultats et analyse les écarts en se mettant à la disposition de la production, pour lui donner les moyens de ses besoins financiers.

\subsection{High-tech}

Les cadres de haut niveau, freinés par les institutions, souhaitent créer des entreprises traitant des techniques de pointe. Leurs qualités de chercheurs, leurs possibilités de raisonnement par analogie les poussent à s'orienter dans des voies à peine ouvertes. Leurs contacts les informent de clientèles guettant ces solutions et prêtes à les payer cher. C'est le monopole de nouveaux marchés pour des années. Ce type d'entreprise est 
considéré comme la "vitrine de la France », important élément du commerce extérieur. La formation d'ingénieur de grande école ou d'université de haut niveau s'affute au long des années par des séminaires de très haut niveau technologique, la lecture et l'analyse des innovations, ainsi que les rencontres internationales. La structure de l'entreprise, autoritaire, admet une certaine autonomie individuelle de recherches et de mises au point après accord du dirigeant sur la validité des projets. Chacun a sa propre organisation, ses matériels souvent conçus et réalisés secrètement. Ce fonctionnement facilite l'épanouissement de tous, les nouveaux produits incitent à l'innovation, la créativité reste en alerte ; le bouillonnement constant des idées fait surgir la réussite ou l'échec - parfois fatal -, mais, toujours, l'expérience de la prochaine entreprise.

Les coûts engendrés doivent être suivis de très près à l'aide de programmes qui tiennent compte des flux financiers espacés mais intenses. C'est une activité trop complexe et spécialisée pour le dirigeant qui la confie à un gestionnaire dont il suit les recommandations qu'il traduit en décision, puis directives pour ses collaborateurs.

Il apparaît, à la fin de cet essai de typologie, que la plupart du temps, le dirigeant de petite entreprise est surtout un technicien, à un niveau - pas forcément scolaire - parfois élevé de sa spécialisation, mais qu'il laisse soit au "hasard", soit à un expert (gestionnaire) le soin de l'équilibre financier de son activité. Or, s'il est vrai que la concurrence internationale se joue sur l'innovation et la qualité, il est non moins certain que l'efficacité des décisions prises, le choix des objectifs et des moyens, peuvent être optimisés par une bonne gestion financière et faciliter ainsi la continuité de l'entreprise.

\section{Se former, ou être formé à la gestion : le dilemme}

L'inintérêt - relatif - porté par la plupart des dirigeants de petites entreprises à l'activité de gestion surprend suffisamment pour qu'on essaye d'en comprendre les raisons: il paraît aussi attirant de pénétrer les rouages de la gestion d'entreprise que ceux d'une nouvelle mécanique ou technique, de décomposer les facteurs d'une prise de décision comme on démonte un matériel pour en déterminer les causes de pannes, ce qui est pratique quotidienne pour un technicien. Pourquoi tant de réticences? Sans doute est-ce dû au manque d'habitude ou à l'ignorance des règles utiles, comme à son conditionnement à une culture trop étroitement technique. Nous nous interrogeons alors sur les raisons de ces réserves vis-à-vis de l'apprentissage de la gestion: où se trouvent les oppositions entre ce type de formation et les aptitudes ou attitudes du dirigeant et, une fois précisées celles-ci, que faire pour les éliminer, et faciliter l'apprentissage?

\section{Disparités entre projet personnel et pratiques de gestion}

La première opposition se situe au niveau de la finalité de l'entreprise. S'y heurtent en effet les espoirs personnels de réussite - surtout subjectifs - et les réalités matérielles de l'existence de l'entreprise. La confrontation porte en elle le risque de mise en cause des convictions en leur donnant un caractère d'illusion.

La deuxième opposition se révèle dans les modalités du fonctionnement: pour le responsable, elles doivent être personnalisées à la mesure de l'entreprise, évoluant avec elle au fil des techniques et au gré des intérêts et satisfactions du dirigeant, courant toute sa vie à la poursuite d'une image en mouvance. Pour la gestion, l'organisme est une entité délimitée par des normes économiques la régissant, et son évolution ne peut se faire qu’à 
travers des schémas préétablis pour ce type d'unité, restreignant les risques d'erreurs et l'imprévu des tentatives.

La troisième opposition porte sur l'organisation des activités : informelle, adaptable, basée à la fois sur l'initiative et l'obéissance stricte de chacun - ce qui est difficilement conciliable - sans échelon hiérarchique intermédiaire responsable. Cette conception du dirigeant se heurte aux structures précisément définies, aux communications écrites et à la rationalisation des activités qui sont la base d'une « saine » gestion d'entreprise.

Enfin la quatrième opposition se fait jour à propos de l'évaluation des résultats: le responsable écoute, estime plus avec subjectivité et intuition que réalisme, comparant l'image de son entreprise (renommée, réputation, conflits avec les clients, le personnel), à celle qu'il avait projetée et, tordant tantôt l'une tantôt l'autre il les met vaille que vaille en concordance. En revanche, les chiffres et ratios établis à partir de mesures de résultats figent dans le bilan une réalité d'évolution et la détermination des actions à venir.

21 Les contradictions inhérentes aux différents modes de direction développés plus haut rendent l'application simultanée de ceux-ci impossible car les décisions s'annuleraient. La synthèse des différentes approches ne peut se faire qu'à travers l'évolution du dirigeant. Encore faut-il qu'il accepte d'entamer une telle démarche lors d'une formation qui lui en ferait éprouver tout l'intérêt et l'inciterait à l'apprentissage d'un autre comportement. Par quel moyen aboutir à ce résultat ? Comment permettre la représentation d'un comportement à base de chiffres et celle de son efficacité pour effacer les craintes qu'il engendre?

\section{Du comportement aux attitudes et aux représentations : une mue difficile}

22 L'activité du gestionnaire met en jeu des pratiques nouvelles, un autre fonctionnement intellectuel, des attitudes inhabituelles au dirigeant de petite entreprise. Il ne s'agit donc pas, dans la formation à la gestion, d'une simple addition aux connaissances du technicien:

\subsection{De l'objet au symbole}

Jusque-là ses actes étaient centrés sur des objets de production; dorénavant il devra en même temps considérer leur évocation chiffrée, contrepartie symbolique du travail à l'aide de signes codifiés. Il ne manipule plus, il suppute; il n'agit plus, il analyse. Ses préoccupations quittent le plan matériel lieu de sa qualification pour un plan imagé, abstrait lieu de sa socialisation. Ce passage, s'il est mal conduit, peut devenir rupture et créer des perturbations dans le comportement habituel du dirigeant.

\subsection{L'apprentissage d'une langue nouvelle}

Nulle symbolisation ne peut être envisagée sans la connaissance des signes dont l'assemblage prend la forme d'un langage, de leur insertion dans la culture de gestion, ainsi que de référentiels utilisables pour mesurer les résultats. Un nouveau vocabulaire où le sens des mots est transposé, où les images suggérées sont totalement étrangères au monde de la production doit être découvert et assimilé ${ }^{2}$. Ce savoir détermine de 
nouveaux systèmes intellectuels, des procédures différentes de décision basées sur la conceptualisation des actes du métier. La maitrise du maniement de ces outils et du champ possible de leur utilisation permet le passage du concret à l'abstrait, capacité indispensable au chef d'entreprise.

\subsection{La conversion identitaire}

Auparavant, le dirigeant regardait l'objet comme une fin, témoin de sa propre qualification et des compétences de l'entreprise. En gestion, l'objet devient un chiffre existant par sa dénomination - produits finis, stock, commande client par exemple infime partie d'une construction numérique où il perd ses caractéristiques propres : son essence est gommée. Ce nouveau regard du dirigeant retentit sur toute sa personne par la nécessité d'identifier ses opinions et attitudes aux normes économiques liées à la culture de gestion, aux comportements rituels des gestionnaires. C'est un groupe auquel il doit appartenir abandonnant celui des techniciens dans une réelle démarche de socialisation.

Nouvelles symboliques, nouveau langage, nouvelle identité, ces facteurs donnent une dimension à la formation, telle, que nous croyons plutôt à un phénomène d'acculturation, dans une perspective de formation/conformation au corps socio-économique auquel le dirigeant va se mêler. Quels supports peuvent permettre cette démarche? Quels médias facilitent la découverte d'une nouvelle culture et le consentement à l'application de ses éléments constitutifs? Quels intermédiaires montrent en surimpression la démarche double à suivre (objets/reflets) avec suffisamment de précision pour motiver à agir dans cette orientation?

\section{A la recherche d'une pédagogie adaptée}

Une telle formation doit correspondre à la personnalité du dirigeant, et aux caractéristiques transculturelles de l'espace dans lequel il évolue.

\subsection{La résistance au formateur}

La formation à la gestion est une des premières manifestations de son autonomie car elle doit, à ses yeux, améliorer sa valeur de dirigeant. Comme la plupart des adultes en formation, il porte en lui la résistance au retour à la situation scolaire, le doute vis-à-vis des connaissances qui sont enseignées. Il veut apprécier de façon perceptible les qualités et l'efficacité de son apprentissage. La forme que prendront ses relations au formateur sera prépondérante dans le niveau atteint. Il y aura :

- affrontement si chacun veut imposer à l'autre ses conceptions, voire son éthique; leur rencontre devient un lieu de combat où chacun n'aura de cesse qu'il ait soumis l'autre à son intellection.

- confrontation si chacun veut exposer à l'autre ses conceptions, voire son éthique; la découverte des oppositions, leur explication permettent à chacun de comprendre la culture de l'autre, d'accepter les différences, d'affermir les concordances. L'accord se fait sur la coexistence, en chacun, d'une part des deux modèles.

- échange si, en situation, chacun communique à l'autre ses conceptions. Recherche des complémentarités, combinaison des éléments contraires permettent à chacun de 
reconstruire son propre cheminement, préférant la codominance à un système dominance/ récessivité. La formation devient efficace comme instrument de changement.

\subsection{Construction identitaire et appropriation} similitudes et tire des conclusions pour les actions à venir. Toute cette activité - même issue d'un savoir livresque - est basée presqu'exclusivement sur l'expérience, la pratique professionnelle acquise un peu en tous sens, au gré des situations. Lorsque la formation est destinée à un dirigeant, le livre, modalité linéaire d'apprentissage n'est pas toujours très adapté : les mots achoppent aux images du réel, aux préoccupations quotidiennes, les lettres figent au contenu. C'est une vertu de l'audio-visuel que de faciliter la formation en rendant l'apprenant prompt à percevoir le sens des événements de l'entreprise.

\section{Dans la lumière des temps}

Entre le commentaire de l'aruspice et l'interactivité des vidéo-disques, de tous temps, les hommes ont souhaité utiliser l'image et le son à des fins pédagogiques. Au deuxième siècle, Horace ouvre un débat qui souligne la primauté de la vue sur l'ouie (art poétique); puis la camera oscura, la lanterne magique au XVII siècle mettent l'image dans les villes et les villages mais c'est au XIX ${ }^{e}$ siècle que, grâce à la photographie, les enseignants utilisent lanternes et clichés pour des projections pendant les cours ${ }^{5}$. Enfin au $\mathrm{XX}^{\mathrm{e}}$ siècle, diapositives, cinéma, vidéo complètent la gamme d'images à vocation éventuelle d'enseignement. L'image est créée à but d'échanges, pour faire partager ce qu'elle 
représente, une réalité concrète ou abstraite. Peu figurative, directive, organisée pour porter un message ou une ambiance, elle peut permettre une certaine autonomie, et donc être un instrument d'individualisation des représentations et d'appropriation de la formation.

Le son est aussi signe de l'existence. Il peut être essentiel, pour représenter un objet, d'en reproduire le son pour approcher de sa réalité. Il touche à la sensibilité, à l'affectif et amplifie les réactions face à l'objet représenté. Le mixage image et son donne et son existence et sa dimension à l'audiovisuel, faisant prévaloir intelligence ou affectivité, réflexe ou sentiment, rationalité ou passion. Bien conçu, bien réalisé, il «crée une expérience globale unifiée. Il s'adresse à l'être tout entier " 6 .

Les divers éléments audio, visuel et audio-visuel permettent la représentation fidèle, ou à effets, des réalités dans des lieux, des espaces, des moments où les phénomènes ne pourraient être mesurés, observés. Quelle que soit la finalité envisagée, l'élément essentiel reste la qualité du message à délivrer et l'activité réceptive de l'apprenant.

L'audio-visuel facilite-t-il l'apprentissage, à travers ses possibilités d'individualisation, de libération (et orientation) de l'imaginaire, d'amplification des représentations, de stimulation de l'activité du récepteur? C'est, nous semble-t-il, une hypothèse à prendre en compte.

\section{Une didactique sur mesure}

37 Pour atteindre les objectifs définis, la formation doit présenter des caractéristiques d'efficacité conçues autour des besoins du dirigeant. Il est en effet essentiel qu'il maîtrise lui-même son acculturation.

\subsection{Le référent de base : l'apprenant}

38 L'appropriation des contenus, parce qu'elle engage son avenir et celui de l'entreprise, est de la responsabilité du dirigeant. Il doit en comprendre les objectifs, en assumer l'apprentissage, en vérifier les résultats. Il pourra mieux y parvenir si les séquences didactiques sont modulaires et courtes: modulaires, c'est-à-dire que chaque séquence comporte un objectif d'apprentissage, une démarche d'acquisition et des aides, un contrôle (autocontrôle) de connaissances, des exercices pratiques d'appropriation, dans une unité de temps, de lieu et d'action. L'ensemble des modules couvre l'ensemble des champs de l'acculturation; courtes, ce qui permet un cadencement rapide des modules et, à la faveur des bons résultats atteints, une réactivation quasi constante des motivations. Par ailleurs, si les résultats ne sont pas favorables, très rapidement l'apprenant peut réagir en rencontrant la personne la plus apte à lui faire rattraper le retard (moindre) pris.

39 Ainsi, en réajustant constamment ses performances aux objectifs à atteindre, prend-il le temps de créer des relations essentielles entre les connaissances à venir et ses expériences et acquis personnels, entre son vécu et son à vivre, bouleversement d'habitudes et de comportements établis. Sa formation est individualisée, elle devient une. 


\subsection{L'influence du milieu socio-économique}

Les pressions conjuguées de la gravité des enjeux et de l'obligation de réussite donnent une importance considérable au niveau de qualité exigé.

De plus, l'intérêt porté aux petites entreprises actuellement, suscite l'apparition de périodiques, magazines, émissions de radio ou de télévision, contrepoints d'une bibliographie considérable, aux thèmes constamment renouvelés. Créations, portraits de dirigeants, exposés des recettes du triomphe ou des causes de l'échec sont le reflet plus ou moins fidèle de ce milieu. Les institutions spécialisées et les experts pullulent, dispensant des conseils. Le responsable peut ainsi méditer sur les analyses d'autrui, confronter ses desseins et ses moyens aux expériences d'autres dirigeants et parfaire ses décisions, si grande est la richesse des matériaux mis à sa disposition. Mais son désarroi peut en être accrû.

\subsection{Le groupe d'apprenants}

2 Autour du dirigeant se retrouvent des compagnons de formation engagés comme lui dans une démarche particulièrement anxiogène. Chacun connaît l'existence des autres, les similarités des projets, des obstacles, des difficultés, l'aide à en tirer, l'appui à leur offrir pour dénouer des situations qui deviendraient fatales. La progression des uns est l'encouragement des autres à travers une sorte de transfert positif qui ancre en chacun l'espoir d'atteindre les objectifs. Mieux que le formateur ils savent entre eux s'expliquer et traduire les mots en termes de vécu, ou extrapoler l'action en symboles, mêlant leurs cultures antécédentes à la culture en cours d'acquisition pour dépasser les difficultés de leur trans-formation.

\subsection{Le formateur-médiateur}

Pour l'apprenant, il est successivement Moi idéal et idéal objectivé

- d'abord parce qu'il représente l'image du gestionnaire en fonctionnement, d'autant plus crédible qu'il est lui-même dirigeant de petite entreprise, investi de la puissance mythique $\mathrm{du}$ formateur; dans le cas contraire, c'est dans l'image collective du groupe que seront puisés les éléments d'une représentation idéale,

- en second lieu parce que sa qualification professionnelle l'autorise, à travers l'évocation des difficultés d'apprentissage à en comprendre les causes et à proposer les éléments de leur dépassement. Cela peut aller du conseil à l'incitation, dans un rôle caractéristique de médiateur. Son efficacité pédagogique sera fonction de la qualité des supports qu'il emploiera pour faciliter les transferts, et de la qualité de leur utilisation.

\section{Du médiateur au média}

L'audio-visuel est un support privilégié pour le dirigeant de petite entreprise, en complément de l'aide apportée par les autres apprenants, le formateur ou des dirigeants qui l'ont précédé, dans son procès d'apprentissage de la gestion. 


\subsection{Une constante disponibilité} pour résultat de permettre au plus large public une utilisation facilitée et rapide des divers instruments: projecteurs de cinéma ou de diapositives, magnétophones, magnétoscopes ne nécessitant en général que quelques manipulations pour le chargement du support, et l'enfoncement d'une ou deux touches pour la mise en route. L'usager peut ainsi, d'une manière aussi fréquente et renouvelée qu'il le souhaite, exploiter les documents mis à sa disposition, aussi aisément qu'il le ferait avec un livre.

\subsection{Le réalisme de la fiction}

Support continuel de la démarche, le document audio-visuel, dans un système interactivé plus évocateur que le livre, renforce l'intérêt de l'apprenant et stimule l'invention par celui-ci des réponses à apporter, à travers les situations proposées. Trois points nous semblent essentiels :

- motiver, inciter à entamer la démarche d'acculturation en dépassant les refus de changement, les craintes du renouvellement. Les spots publicitaires sont le modèle de ce type de documents, imposant une image positive de l'inconnu ;

- construire de nouvelles attitudes en prenant pour point de départ ceux qui ont réussi dans cette même voie, en tentant de reproduire tout ou partie de leur démarche pour, ensuite, l'évaluer et se l'approprier. Portraits et reportages apportent des matériaux de fond à cette restructuration;

- apprécier l'efficacité des modifications de comportement à travers des résultats atteints ou simulés. Les fictions à choix multiples de solutions soutiennent activement cette démarche.

Ces objectifs seront d'autant mieux atteints qu'aura été choisi par le concepteur audiovisuel, le «bon langage » pour ce type d'enseignement. Il n'apparaît cependant pas encore fréquemment un langage de fiction dans le domaine de l'enseignement de la gestion.

\subsection{Un espace transitionnel}

Jamais pourtant, des images et des sons, aussi bien mixés soient-ils, n'ont atteint leur but pédagogique par leurs seules vertus d'images et sons. Leur rigidité éventuelle ennuie, lasse, éloigne ; c'est le cas d'un grand nombre de films documentaires : ils ne laissent aucune place à l'imaginaire du récepteur, à sa singularité. Ils ne sont pas un «espace neutre, lieu de repos pour l'individu engagé dans cette tâche humaine incessante qui consiste à maintenir la réalité intérieure et la réalité extérieure distinctes, et néanmoins étroitement en liaison " ${ }^{7}$.

L'audio-visuel en apprentissage de la gestion peut être cet espace quand il est convenablement conçu. Sa puissance évocatrice, sa formidable capacité à déclencher l'imaginaire, facilitent pour l'apprenant la sauvegarde d'un lieu où s'élaborent ses attitudes et comportements à venir, où il les confronte à ceux que d'autres dirigeants ont eus dans des situations analogues. Où il en vérifie la validité et en décide l'appropriation.

C'est à cette dimension que se reconnaît un audio-visuel de formation transculturelle. 


\section{BIBLIOGRAPHIE}

Auvolat, J.M., Lavigne, J.C. \& Mayère, A., (1985), « L'artisanat en France, réflexion générale et prospective ", Paris, Éd. La Documentation Française, p. 254.

Babin, P. \& Kouloumdjian, M.F., (1983), Les nouveaux modes de comprendre, la génération de l'audiovisuel et de l'ordinateur, Paris, Éd. du Centurion.

Bianchi, J. \& Kouloumdjian, M.F., (1986), L'espace social de la communication, Écully, CNRS/ IRPEACS.

Linton, Ralph, (1965), Le fondement culturel de la personnalité, traduit par A. Lyotara avec une préface de J.C. Filiaux, Paris, Dunod.

Thollon-Pommerol, C., (1983), Pédagogie audio-visuelle : histoire et actualité, Genève, Université de Genève, Faculté-de Psychologie et des Sciences de l'Éducation, $\mathrm{n}^{\circ} 35$.

Winnicott, D.W., (1975), Jeu et Réalité, Paris, Gallimard.

\section{NOTES}

1. J.M. Auvolat, J.C. Lavigne \& A. Mayère, «L'artisanat en France, réflexion générale et prospective », Paris, Éd. La Documentation Française, 1985, p. 254.

2. «Les immobilisations c'est de l'actif » oblige le dirigeant de petite entreprise à oublier le sens donné aux mots jusque-là, mais aussi à en déduire une attitude opposée à celle qu'il aurait eue puisque de négative (immobilisations) elle devient positive (actif).

3. Ralph Linton, Le fondement culturel de la personnalité, traduit par A. Lyotara avec une préface de J.C. Filiaux, Paris, Dunod, 1965.

4. Cf. le concept d'appropriation, J. Bianchi \& M.F. Kouloumdjian, L'espace social de la communication, Écully, CNRS/IRPEACS, 1986.

5. Cf. Thollon-Pommerol, Pédagogie audio-visuelle: histoire et actualité, Genève, Université de Genève, Faculté-de Psychologie et des Sciences de l'Éducation, $n^{\circ} 35$.

6. P. Babin \& M.F. Kouloumdjian, Les nouveaux modes de comprendre, la génération de l'audio-visuel et de l'ordinateur, Paris, Éd. du Centurion, 1983.

7. D.W. Winnicott, Jeu et Réalité, Paris, Gallimard, 1975.

\section{RÉSUMÉS}

La PME est, ici, définie comme une entreprise où le le dirigeant participe aux activités de production. Cela n'empêche pas que celui-ci doit assurer la gestion, notamment financière, de son entreprise. Après avoir brossé quatre portraits-types de dirigeants de PME l'auteur se demande pourquoi ceux-ci se désintéressent tant de la gestion. Cette problématique relève, en 
fait, d'un problème pédagogique et du domaine de la formation et de l'apprentissage. Il s'agit alors de penser une pédagogie nouvelle pour les formations professionnelles dédiées à ce type d'individus. Pour l'auteur l'audiovisuel peut faciliter l'apprentissage de la gestion grâce à sa force d'illustration.

INDEX

Keywords : vocationnal training, business manager, SME, financial management, audiovisual, learning

Mots-clés : formation professionnelle, chef d'entreprise, PME, gestion financière, audiovisuel, apprentissage

\section{AUTEURS}

\section{MARIE-FRANCE KOULOUMDJIAN}

Marie-France Kouloumdjian, Université de Lyon 1/CNRS-IRPEACS

LOUIS ARMELLINO

Louis Armellino, Université de Lyon 1/CNRS-IRPEACS 